\title{
A ROBUST BAND-TRACKING PACKET DETECTOR (BT-PD) IN OFDM-BASED ULTRA-WIDEBAND SYSTEMS
}

\author{
Jyh-Ting (Justin) Lai, Chun-Yuan Chu, An-Yeu (Andy) Wu, and Wen-Chiang Chen*
}

\author{
Graduate Institute of Electronics Engineering, and \\ Department of Electrical Engineering, National Taiwan University \\ *Faraday Technology Corporation
}

\begin{abstract}
Multiband orthogonal frequency-division multiplexing (MB-OFDM) systems employ frequencyhopping technology to achieve the capabilities of multiple access and frequency diversity. However, they also complicate packet detector (PD) and time-frequency code synchronization, in terms of the requirement for fast synchronization for the frequency hopping, the extremely low receiver sensitivity, and the high hardware complexity. In this paper, we firstly systematically analyze the differences between MB-OFDM and conventional OFDM systems, and then propose a band tracking PD (BT-PD) that can cope with a worse-case multipath channel SNR of $-8.4 \mathrm{~dB}$ with a packet detection error rate of less than $10^{-5}$.

\section{INTRODUCTION}

Multiband orthogonal frequency division multiplexing (MB-OFDM) technology (or so-called timefrequency interleaved OFDM) has recently been applied to wireless personal area networks [1][2]. This technique increases both the traffic capacity and the frequency diversity, and it follows the relevant Federal Communications Commission (FCC) regulations, which specify that the $7500 \mathrm{MHz}$ spectrum can be used for unlicensed ultra-wideband communication devices. Furthermore, MB-OFDM employs several types of timefrequency code (TFC) in the preamble part to easily identify the packet properties and frequency-hopping sequences. However, even though MB-OFDM can lever the successful experience of other OFDM systems, such as wireless local area network (WLAN) [3] and digital video broadcasting terrestrial TV [4], there are still many differences between MB-OFDM and conventional OFDM systems, and these differences are often the bottlenecks of system performance. The major bottleneck of the receiver front-end is the requirement of high noise immunity. For MB-OFDM system, to avoid interfering with existing services for other communication systems, the power spectral density must not exceed $-41.25 \mathrm{dBm}$ in a $1-\mathrm{MHz}$ bandwidth [1]. The power spectral density of the interference at the receiver of the MB-OFDM system could be $10^{7}$ higher than that of the MB-OFDM signal.
\end{abstract}

Therefore, the noise immunity capability in the MBOFDM receiver should be higher than that of existing commercial OFDM systems.

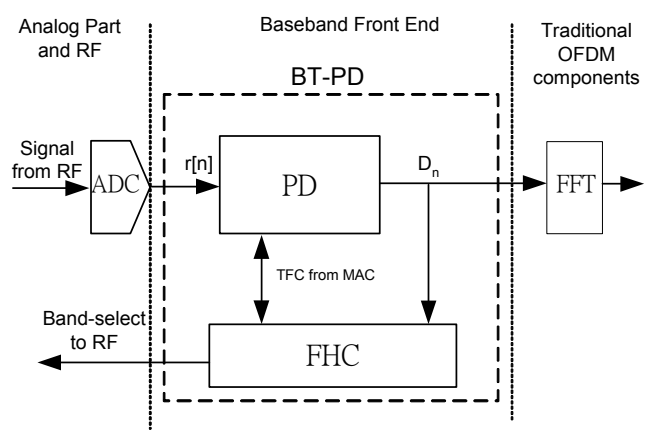

Fig. 1 Block diagram of the proposed BT-PD.

In this paper we systematically analyze the challenges of the MB-OFDM system, focusing on the baseband front-end circuits shown in the dashed box of Fig. 1. To address the above-mentioned difficulties, we propose a combined solution - called the band-tracking PD (BT-PD) algorithm - that includes both performance and hardware improvements. The details of the proposed BT-PD are shown in Fig.1, where the functions of the building blocks are as follows:

- PD: The PD is the datapath circuit that quantifies the strength of input signal in each band. The design goal of the PD is to maximize the signal-to-noise ratio (SNR) whilst minimizing the gate count of hardware.

- Frequency-Hopping Control: The frequency-hopping control (FHC) circuit controls the RF circuit to receive signals on the correct bands following the instruction from the PD.

To overcome the difficulties of the above components, we propose a series of solutions in our baseband front-end, named the BT-PD. It can not only cope with the frequency-hopping environment by actively tracking the receiving band, but also requires very little hardware. The BT-PD can operate well in an SNR of -8.4 $\mathrm{dB}$ with packet detection error rate (PDER) of less than $10^{-5}$ in the channel with the worse-case fading. 


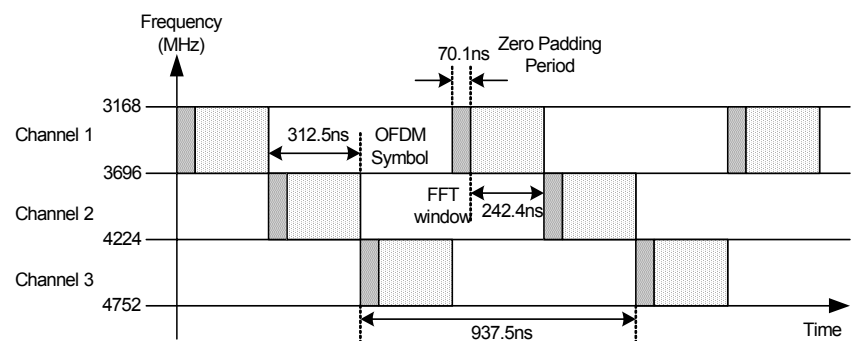

Fig. 2. Hopping diagram for the MB-OFDM system

\section{MB-OFDM DESIGN ISSUES IN THE BASEBAND FRONT-END}

1) Frequency hopping for adjacent symbols: From Fig. 2, each OFDM symbol, including the packet preamble, will be transmitted on specific bands as determined by given TFC. We have to redesign the PD to detect packets in accordance with this protocol. In addition, to highlight the problems of a conventional PD, we plot the architecture of a conventional PD apparatus [5] in Fig. 3. The lower branch (containing a squarer) calculates the input signal power, and the upper branch (containing a multiplier) calculates the signal correlation power between adjacent symbols. If the received signal comprises only noise, the power ratio $M_{n}$ is 0 because the output power of the cross-correlation function between adjacent symbols is almost zero. Otherwise, if the received signal is the preamble part of the packet, the output of the crosscorrelation function between adjacent symbols is almost the same as that of the auto-correlation function of the preamble part, so the power ratio $M_{n}$ is much larger. Fig. 4 shows the output waveform of the power ratio. We can set the threshold value between 0 and 1 for determining whether a packet has been received. However, using the correlation power is no longer suitable for the MB-OFDM system because the adjacent symbols are conveyed in different bands whereas the received band is fixed before the packet is detected.

2) Large dynamic range of the received power and the SNR: Unlike a WLAN application where the PD can be truly active after the automatic gain control (AGC) and variable-gain amplifier (VGA) have stabilized, the $\mathrm{PD}$ in some MB-OFDM receivers must be active before the AGC/VGA is stable in order to correctly assign the RF signal to the next receiving band. For this architecture, the PD should work well over a power range of more than $60 \mathrm{~dB}$, even if the ADC is saturated. In addition, because the input SNR is from 24 to $-8.4 \mathrm{~dB}$ as mentioned above, the large dynamic ranges of the input power and the SNR make designing the PD difficult. Indeed, none of the existing solutions [5]-[7] can cope with this problem.

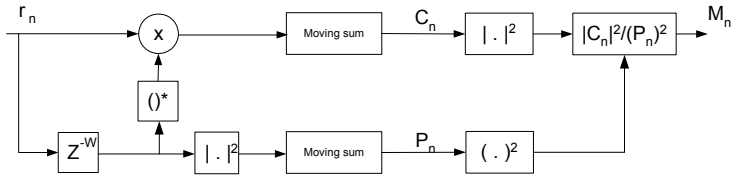

Fig. 3 A traditional PD.

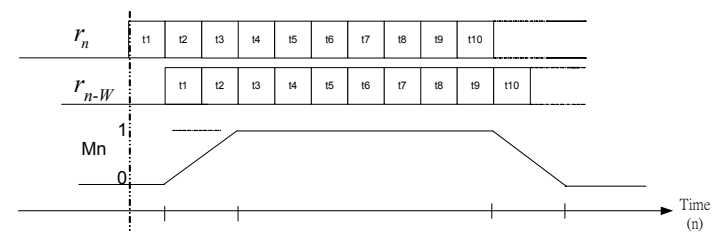

Fig. 4 Symbol correlation output of a traditional PD.

\section{THE PD OF THE PROPOSED BT-PD ALGORITHM}

The proposed BT-PD comprises the 2 major components shown in Fig. 1: the PD and FHC. In this section we introduce the $\mathrm{PD}$ and its subcomponents: symbol combiner (SC), symbol detector (SD), and channel length indicator (CLI). The algorithm derivation of the the FHC is discussed in Section 4.

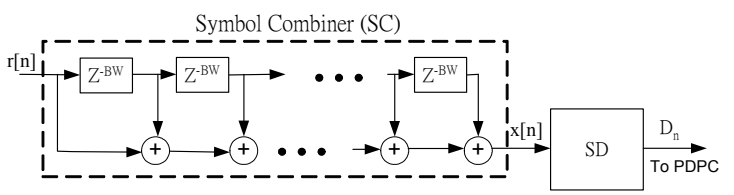

(a)

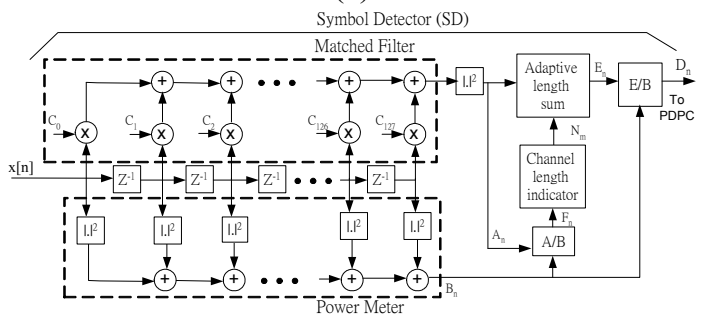

(b)

Fig. 5 Datapath block diagrams of the BT-PD: (a) The SC and $\mathrm{SD}$ in the PD for $T F C=1$; (b) detailed circuit of the SD.

\subsection{SC}

Block diagrams of the SC and SD are shown in Fig. 5(a) and (b), respectively. In order to increase the noise immunity, we use an SC to combine several preamble symbols - instead of using only one symbol - to detect the packet arrival. Because the preamble symbols are repeated, this technique will increase the effective SNR. However, because the RF circuit for receiving is fixed at a specific band before the PDPC formally declares that a packet has arrived, the received signal only appears in the time slot when the transmitted band is the same as the (fixed) receiving band. Therefore, we have to collect signal 
samples in a queue and sum the suitable time slot when there are signals, such as [1]:

$$
\begin{array}{ll}
x(n)=\sum_{k=0}^{J-1} r(n-B W k) & (\text { for } T F C=1,2) \\
x(n)=\sum_{k=0}^{J-1} r\left(n-2 B W\lfloor k / 2\rfloor+W((k))_{2}\right), & (\text { for } T F C=3,4) \\
x(n)=\sum_{k=0}^{J-1} r(n-W k) & (\text { for } T F C=5,6)
\end{array}
$$

where $r(n)$ is the received signal in the baseband, $J$ is the number of combined symbols, $B$ is the total number of bands in the band group, $W$ is the total number of samples in one OFDM symbol, " $\lfloor\rfloor$.$" denotes the round-to-floor$ operation, " $((.))_{2}$ " denotes the base- 2 remainder operation, and

$$
W=N+L,
$$

where $N$ is the FFT window size and $L$ is the zero-padding duration. Typical values of $B, N$, and $L$ are 3,128 , and 37 , respectively. From Eq. (1), the maximal length of the input queue is

$$
G=(J-1) B W+1 .
$$

The SC can improve the received SNR by $J$ times. In addition, because the storage devices of the input queue can be shared with other components, such as the sampling frequency offset compensation circuit or the frame synchronization circuit, there is no extra loading for the memory devices in the SC.

\subsection{SD}

The proposed SD structure is shown in Fig. 5(b). The upper block in the figure is the matched filter, which is an FIR filter with preamble coefficients as filter coefficients, and the lower block is the power meter, which provides the power sum of tap data. Before deriving the algorithm, we explain the basic properties of the preamble coefficients of the MB-OFDM system. We define the coefficient vector as

$$
\mathbf{c} \equiv\left[c_{0}, c_{1}, \cdots, c_{N-1}\right]^{\mathrm{T}},
$$

where the $c_{i}$ variables are the preamble coefficients. The inner product of the coefficient vector is normalized as [1]

$$
\mathbf{c}^{\mathrm{H}} \mathbf{C}=N \text {. }
$$

Because zero-padding signaling is adopted in the MBOFDM system, the transmitted OFDM symbol can be expressed as the following vector form:

$$
\begin{aligned}
& \mathbf{s}(n)=[s(n), s(n+1), \cdots, s(n+W-1)]^{\mathrm{T}}, \\
& s(n)=\left\{\begin{array}{cc}
c_{((n))_{W}}, \text { if }((n))_{W}<N \\
0, & \text { otherwise }
\end{array}\right.
\end{aligned}
$$

where $((n))_{W}$ denotes the remainder of $n$ divided by $W$. From the quasi-orthogonal properties of the preamble coefficients, the cross-correlation between the coefficient vector and transmitted symbol vector is

$$
\left[\mathbf{c}^{\mathrm{T}}, \mathbf{0}_{L}^{\mathrm{T}}\right]^{\mathrm{T}} \mathbf{s}(n)=\delta(\kappa)+v(\kappa) \cong \delta(\kappa)
$$

and

$$
\kappa \equiv((n))_{W},
$$

where $\delta($.$) is the delta function, \mathbf{0}_{L}$ is the zero vector with length $L$, and $v($.$) is the small sidelobe signal which can be$ neglected because $\|\delta(0)\|^{2}>\|v(\kappa)\|^{2}$ for all $\kappa$.

On the receiver side we assume there are $J$ symbols combined together in the SC, as shown in Fig. 5(a). To simplify the derivation, we assume that the CFO is small, so the received signal of the preamble part can be expressed in the following matrix form [9]:

$$
\begin{aligned}
& \mathbf{x}(n)=J \mathbf{S}(n) \mathbf{h}+\sum_{k=0}^{J-1} \mathbf{n}_{k}(n), \\
& \mathbf{x}(n)=[x(n), x(n+1), \cdots, x(n+W-1)]^{\mathrm{T}}, \\
& \mathbf{h}=\left[h_{0}, h_{1}, h_{2}, \cdots, h_{N_{m}-1}\right]^{\mathrm{T}}, \\
& \mathbf{S}(n)=\left[\mathbf{s}(n), \mathbf{s}(n-1), \cdots, \mathbf{s}\left(n-N_{m}+1\right)\right]^{\mathrm{T}},
\end{aligned}
$$

where $\left\{h_{l}\right\}$ denotes the normalized CIR, $N_{m}$ is the channel duration, and $\mathbf{n}_{k}(n)$ is the identical independent distribution (i.i.d.) noise vector with power $\sigma_{n}^{2}$. Substituting Eq. (10) into Eq. (12) and assuming that the received signal power is $\sigma_{s}^{2}$ yields that the matched-filter output divided by $J N \sigma_{s}$ is the estimator of the normalized CIR:

$$
\frac{1}{J N \sigma_{s}} \mathbf{c}^{H} \mathbf{x}(n)=\hat{h}_{k}+\mathbf{c}^{H} \sum_{k=0}^{J-1} n_{k}(n) \cdot
$$

The output power $A_{n}$ of the matched filter is

$$
A_{n}=\mathbf{c}^{H} \mathbf{x}(n) \mathbf{x}^{H}(n) \mathbf{c} .
$$

Substituting Eq. (16) into Eq. (17) and taking the expectation value yields

$$
\begin{gathered}
\widetilde{A}_{n} \equiv E\left[A_{n}\right]=\mathbf{C}^{H} E\left[\mathbf{X}(n) \mathbf{X}^{H}(n)\right] \mathbf{C} \\
=J^{2} N^{2} \sigma_{s}^{2}\left|\hat{h}_{\kappa}\right|^{2}+J N \sigma_{n}^{2}
\end{gathered}
$$

Therefore, the expectation value of the average sum output is

$$
\widetilde{E}_{n} \equiv E\left[E_{n}\right]=\sum_{k=n}^{n+N_{m}-1} \widetilde{A}_{k}=J^{2} N^{2} \sigma_{s}^{2}+J N_{m} N \sigma_{n}^{2},
$$

where we use the property of the normalized channel, that is,

$$
\sum_{\kappa=0}^{N_{m}-1}\left|h_{\kappa}\right|^{2}=1
$$

and assume that $N_{m}$ is known and can be obtained from the CLI. The derivation of $N_{m}$ will be explained in Section 3.3. Next, the power-meter output $B_{n}$ and its expectation value are

$$
B_{n}=\mathbf{x}^{\mathrm{H}}(n) \mathbf{x}(n),
$$

and

$$
\widetilde{B}_{n} \equiv E\left[B_{n}\right]=E\left[\mathbf{X}(n)^{\mathrm{H}} \mathbf{X}(n)\right]=N \sigma_{x}^{2}=N\left(J^{2} \sigma_{s}^{2}+J \sigma_{n}^{2}\right) .
$$

If a signal is not received or the signal in the matched filter does not match the coefficient vector (i.e. $\sigma_{s}^{2}=0$ ), the expectation value of the filter output can be derived from Eqs. (18) and (22) as

$$
\widetilde{D}_{n} \equiv E\left[D_{n}\right]=\frac{\widetilde{E}_{n}}{\widetilde{B}_{n}}=\frac{J N_{m} N \sigma_{n}^{2}}{J N \sigma_{x}^{2}}=N_{m} \cdot
$$

Otherwise, if the $\mathrm{SD}$ receives a preamble signal with correct timing, the matched-filter output is 


$$
\tilde{D}_{n}=\frac{J^{2} N^{2} \sigma_{s}^{2}+J N_{m} N \sigma_{n}^{2}}{N\left(J^{2} \sigma_{s}^{2}+J \sigma_{n}^{2}\right)}=\frac{J N \sigma_{s}^{2}+N_{m} \sigma_{n}^{2}}{J \sigma_{s}^{2}+\sigma_{n}^{2}}
$$

To provide an intuitive understanding, we consider two extreme cases. First, for very high SNR (i.e., $\sigma_{s}^{2}>>\sigma_{n}^{2}$ ) the SD output is bounded by

$$
\widetilde{D}_{n} \approx N
$$

Second, for very low SNR (i.e., $\sigma_{s}^{2}<<\sigma_{n}^{2}$ ) the PD output is

$$
\widetilde{D}_{n} \approx J N \cdot S N R+N_{m} \cdot
$$

The SD output is connected to the FHC, and we can set a threshold level $\eta$ in the FHC. If the SD output $D_{n}$ is larger than the level, this means that a packet has arrived. The properties of the proposed SD are as follows:

1. The SD can operate well in poor-SNR conditions. In contrast to a traditional PD, which fails in the low-SNR case, our SD in the BT-PD will increase the input sensitivity $J N$ times in the poor-SNR condition.

2. From the extreme case of Eqs. (25) and (26), the output is bounded from $N$ to $N_{m}$, so the threshold level is independent of the input power.

3. The division E/B in Eq.(24) and in Fig. 5 can be avoided simply by checking the following inequality:

$$
E_{n} \geq B_{n} \cdot \eta,
$$

where $\eta$ is the threshold level.

4 For the $k^{\text {th }}$ preamble symbol, the best symbol window $\mathbf{X}\left(n_{0}\right)$ can be obtained from the SD output from

$$
\left\{\mathbf{X}\left(n_{0}\right) \mid n_{0}=\arg \max _{n}\left(D_{n}\right), k W<n \leq(k+1) W\right\} .
$$

In addition, the best FFT window can also be obtained from

$$
\left\{\mathbf{X}^{\prime}\left(n_{1}\right) \mid n_{1}=\arg \max _{n}\left(A_{n}\right), k W<n \leq(k+1) W\right\},
$$

where

$$
X^{\prime}\left(n_{1}\right) \equiv\left[x\left(n_{1}\right), x\left(n_{1}-1\right), \cdots, x\left(n_{1}-N+1\right)\right]^{T} .
$$

Therefore, the SD provides the symbol synchronization information to circuitry of the inner receiver such as the FFT and OLA circuits, and the equalizer.

5. The power meter in the BT-PD can be used for other purposes. For example, in the clear-channel assignment (CCA) process, the power meter can provide the input power value to calculate the gain of the AGC/VGA.

\subsection{Run-time CLI}

In Eqs. (18) and (19), we assume that the channel length $N_{m}$ is known in advance. However, it is not true in reality. If the $N_{m}$ is too small, the energy in each multipath channel cannot be collected and the performance of the SD will be degraded. On the other hand, if $N_{m}$ is too large, the noise term in Eq. (24) will also degrade the SD sensitivity. Our goal here is to propose a run-time CLI that can estimate the channel length and provide it to the SD. From [1], if we neglect the ray distribution, the CIR and the distribution of the cluster arrival time are given by

$$
\begin{gathered}
h(t)=\sum_{l=0}^{V-1} \alpha_{l} \delta\left(t-T_{l}\right), \\
p\left(T_{l} \mid T_{l-1}\right)=\Lambda \exp \left(-\Lambda\left(T_{l}-T_{l-1}\right)\right), l>0,
\end{gathered}
$$

where the property of $\alpha_{l}$ is

$$
E\left[\left|\alpha_{l}\right|^{2}\right]=\Omega_{0} \exp \left(-T_{l} / \Gamma\right)
$$

where $\Gamma$ is the RMS delay of the CIR, $V$ is the number of multipaths, $\Lambda$ is the cluster arrival rate, $\left\{T_{l}\right\}$ is the delay of the $l^{\text {th }}$ cluster, and $\Omega_{0}$ is the initial cluster power. Assuming that the sampling period is $T_{0}$, and substituting Eqs. (32), (33), and (20) into Eq. (31), the power of the normalized channel is

$$
\begin{gathered}
E\left\{\left|h_{\kappa}\right|^{2}\right\}=\left(1-\exp \left(-T_{0} / \Gamma\right)\right) \exp \left(-\kappa T_{0} / \Gamma\right), \\
\cong\left(1-\exp \left(-3 / N_{m}\right)\right) \exp \left(-3 \kappa / N_{m}\right)
\end{gathered}
$$

where $\kappa$ is defined in Eq. (11) and we assume that $N_{m}$ is 3 times the sampled RMS delay $\Gamma / T_{0}$. For this condition, $95 \%$ of the channel energy will be included within 0 to $N_{m}-1$ taps. Next, from Eq. (34) and applying a Taylor expansion, the maximal value of the estimated CIR can be replaced by $N_{m}$ as follows:

$$
\max _{\kappa} E\left\{\left|h_{\kappa}\right|^{2}\right\}=\left(1-\exp \left(-3 / N_{m}\right)\right) \cong 3 / N_{m} \text {. }
$$

To estimate the channel length, we define parameter $F_{k}$ as $A_{k} / B_{k}$, and from Eqs. (18) and (22) the expectation value of $F_{k}$ is

$$
\widetilde{F}_{n} \equiv E\left[F_{n}\right]=\frac{\widetilde{A}_{n}}{\widetilde{B}_{n}}=\frac{J N \sigma_{s}^{2}\left|h_{\kappa}\right|^{2}+\sigma_{n}^{2}}{\sigma_{s}^{2}+\sigma_{n}^{2}} .
$$

We neglect the noise term $\sigma_{n}^{2}$ in Eq. (36) and substitute Eq. (35) into Eq. (36) using $F_{n}$ instead of $\widetilde{F}_{n}$, which yields the estimated channel length:

$$
N_{m} \cong 3 J N / \max _{n} F_{n} \text {. }
$$

The optimal SD output can be obtained by substituting the value of $N_{m}$ into the average sum circuit or Eq. (19).

\section{SIMULATION RESULTS AND PERFORMANCE ANALYSIS}

\subsection{Simulation environment and specification}

The specification in [1] defines that the start of a valid OFDM transmission at a receiver level of at least $83.5 \mathrm{dBm}$ (minimum sensitivity at the lowest information data rate of $53.3 \mathrm{Mb} / \mathrm{s}$ ) should cause CCA or the PD to declare "busy" with a probability greater than $90 \%$ within $4.6875 \mu \mathrm{s}$. In the system simulation, the SNR was $-5.5 \mathrm{~dB}$ at the lowest information data rate. To implement a more robust receiver in the receiver front-end, the PD system should operate well at $3 \mathrm{~dB}$ below the lowest information data rate. Hence, our target SNR is $-8.4 \mathrm{~dB}$, with packetloss and false-alarm probabilities being less than $10^{-5}$.

\subsection{Simulation of the BT-PD operation}

The SNR range of the BT-PD simulation was from 24.0 to $-9.4 \mathrm{~dB}$ with some nonideal effects such as the noise figure, channel fading, frequency, and phase offset. The detailed behavior of the BT-PD is provided in Fig. 6 . 
Fig. 6(a) shows the received power waveform with SNR = $0 \mathrm{~dB}$. It indicates that the starting point of the signal is blurred by the high noise power. Fig. 6(b) shows the transmitting and receiving bands for each OFDM symbol. Both lines are very close, indicating that our approach can correctly trace the transmitting bands even though the receiver suffers from multipath fading. Fig. 6(c) is the SD output waveform, where the peak values for different OFDM symbols differ because each has different timing, phase, and noise values. Therefore, our FHC state machine in the BT-PD has to keep the maximum output of the SD (the dashed line) and indicate the best FFT window and best timing to the frame synchronization circuit. Fig. 6(d) and (e) are the symbol counter and sample counter, respectively. The symbol counter counts the symbol index from the first recognized preamble symbol and tells the system what the symbol means (i.e., preamble or data). The sample counter indicates the index of the current sample in this OFDM symbol.

The simulation results indicate that the BT-PD can correctly instruct the RF circuit to track the transmitted bands and symbols in a channel with a poor SNR.
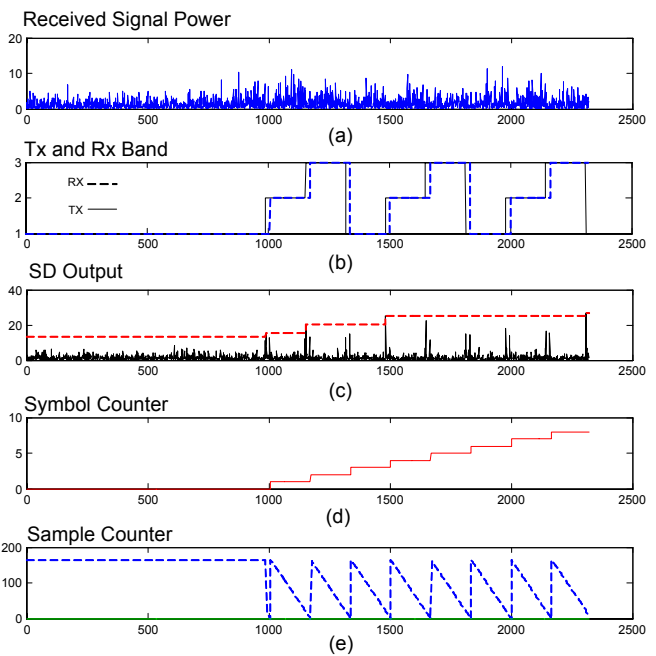

Fig. 6 Diagrams of the operation of the BT-PD: (a) received signal power $(S N R=0 \mathrm{~dB})$, (b) transmit and receive bands, (c) SD output waveform, (d) symbol-

counter index, and (e) sample-counter index.

\subsection{Performance measures}

We define two measures to evaluate the performance of the PD system: the maximal correctly receiving probability (MCRP), which is given by

$$
M C R P \equiv \max _{\eta}\left\{\mathbf{P}_{\text {Normal }}(\eta)\right\} \text {; }
$$

and the threshold range (TR) for which more than $90 \%$ packets are correctly received, which is given by

$$
T R \equiv \max \{\eta\}-\min \{\eta\} \text { for }\left\{\eta \mid \mathbf{P}_{\text {Normal }}(\eta) \geq 0.9\right\} \text {. }
$$

From the MB-OFDM specification in Section 4.1, the MCRP should be higher than $10^{-5}$. However, the MCRP cannot really reflect the performance when its value is very close to 1 because for this case we have to simulate a huge number of packets to detect one that is received incorrectly in order to calculate the MCRP. It may therefore be time consuming to evaluate the probabilities for the full range of the threshold level. In contrast, the TR is easily obtained after only several hundred packet realizations. Moreover, the TR will change with any minor modifications to the system, and hence this parameter therefore provides an easy method of determining detailed improvements and degradations resulting from even minor changes in the PD system.

\subsection{Performance comparison}

4.4.1 System performance of an existing solution

The performance simulations of the existing solution [8] are shown by the three curves in Fig. 7 of the probabilities defined as follows:

1. $\boldsymbol{P}_{\text {Normal }}$ : the probability of the PD system correctly detecting a packet within $4.6875 \mu$ s from the beginning of a packet arrival.

2. $\boldsymbol{P}_{\text {Loss: }}$ : the probability of the PD system not detecting a packet within $4.6875 \mu$ s from the beginning of a packet arrival.

3. $\boldsymbol{P}_{F A}$ : the probability of the PD system declaring "busy" when there is no packet present.

For each point in Fig. 7, we ran $10^{6}$ simulations for different thresholds with different noise seeds and random timing offsets. The SNR was $-8.4 \mathrm{~dB}$, while the CM4 multipath channel was used. The figure shows that the MCRP is only 0.47 at threshold level 16 , indicating that the performance does not meet the specification described in Section 4.1. In addition, because $\boldsymbol{P}_{\text {Normal }}$ is never higher than $90 \%$ for each threshold level, the TR is 0 .

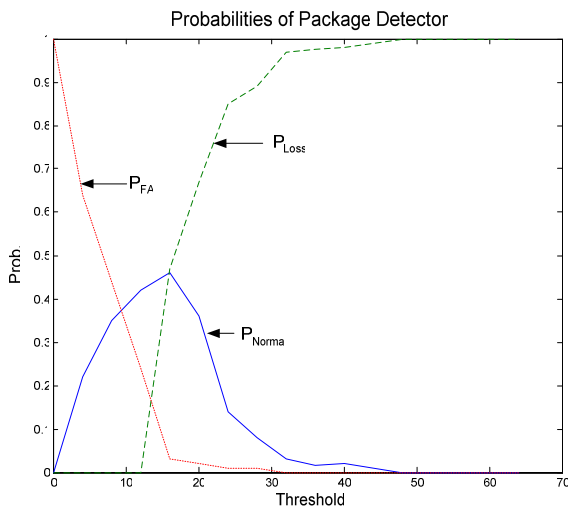

Fig. 7 Performance of the existing PD for the CM4 channel with $S N R=-8.4$.

\subsubsection{System performance of the proposed BT-PD}

The performance of the proposed BT-PD is shown in Fig. 8, in which there are 3 curves for each of $\boldsymbol{P}_{\text {normal }}, \boldsymbol{P}_{F A}$, and $\boldsymbol{P}_{\text {Loss }}$ corresponding to 3 SNR values $(-7.4,-8.4$, and - 
$9.4 \mathrm{~dB}$ ), and where the CM4 multipath channel was used. Fig. 8 indicates that the suitable threshold range will be low with a poor SNR, but that there is still a large range of the threshold level at which $\boldsymbol{P}_{\text {Loss }}$ and $\boldsymbol{P}_{F A}$ are all sufficiently low. For our target SNR $(-8.4 \mathrm{~dB})$ the TR is 18.2, and the probability curves are replotted in Fig. 9 on a logarithmic scale. This figure indicates that for a threshold level of 22 , the MCRP is almost $100 \%$ (or, say, more than $10^{-5}$ in the restrictive sense). To sum up, our design can declare "busy" correctly within $4.6875 \mu$ s at the threshold range from 18 to 24 under the worse-case multipath channel, with both $\boldsymbol{P}_{\text {Loss }}$ and $\boldsymbol{P}_{F A}$ (or the $\boldsymbol{P}_{\boldsymbol{P D E R}}$ ) being less than $10^{-5}$. This result is superior to that for the restrictive specification.

In Table 1, we compare the performances for several existing PD solutions. The simulation conditions are the same as for Fig. 9. Because there is no power meter in [8] and the PD cannot operate normally without using AGC, we assume that the AGC is ideal in our simulation. In contrast, the tap-decrease method in [10] reduces the taps of the matched filter, so the performance is degraded relative to [8]. Table 1 indicates that the proposed BT-PD algorithm is superior to other existing approaches.

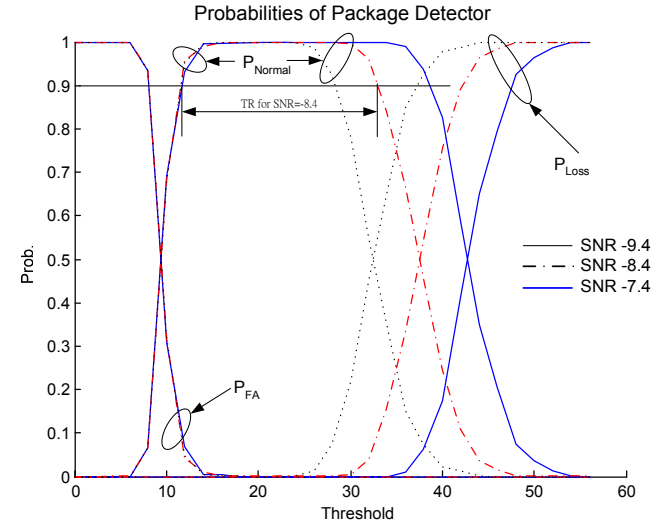

Fig. 8 The BT-PD performance in the CM4 channel for $S N R=-7.4,-8.4$, and $-9.4 \mathrm{~dB}$.

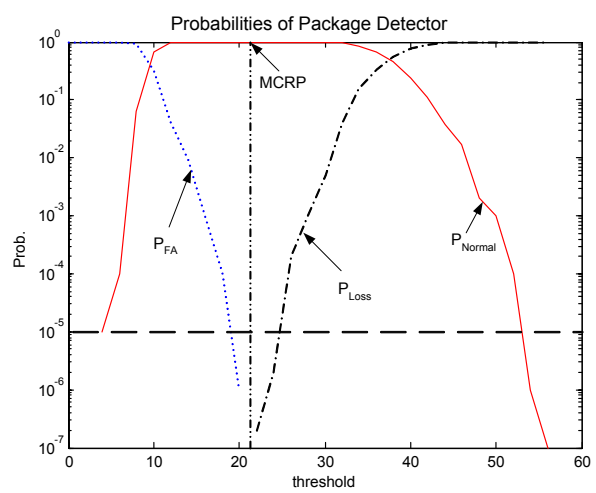

Fig. 9 The BT-PD performance and the MCRP for $S N R=-8.4$.

\begin{tabular}{|c||c|c|c|}
\hline $\begin{array}{c}\text { Scheme } \\
\text { Measure }\end{array}$ & $\begin{array}{c}\text { The PD } \\
\text { in [8] }\end{array}$ & $\begin{array}{c}\text { Tap-decrease } \\
\text { method in [10] }\end{array}$ & BT-PD \\
\hline \hline MCRP & $47 \%$ & $30 \%$ & $>1-10^{-5}$ \\
\hline TR & 0 & 0 & 18.2 \\
\hline
\end{tabular}

Table 1 Performance comparisons of different PD schemes.

\section{CONCLUSIONS}

The PD of a MB-OFDM system is very difficult to design because of the requirement for frequency hopping and the hardware complexity. In addition, it should be able to cope with an SNR as low as $-8.4 \mathrm{~dB}$. Moreover, the BT-PD can cope with a worst-case SNR with a PDER of less than $10^{-5}$.

\section{REFERENCES}

[1] A. Batra et al., "Multiband OFDM physical layer proposal for IEEE 802.15 Task Group 3a," Sept. 14, 2004.

[2] A. Batra et al., "Design of a multiband OFDM system for realistic UWB channel environments," IEEE Trans. Microwave Theory and Techniques, vol. 52, pp. 2123-2138, Sept. 2004.

[3] IEEE 802.11a Standard, ISO/IEC 8802-11: 1999/Amd 1:2000(E).

[4] "Digital broadcasting systems for television, sound and data services; framing structure, channel coding and modulation for digital terrestrial television," May 1996.

[5] J. Terry and J. Heiskala, OFDM Wireless LANs: A Theoretical and Practical Guide $2^{\text {nd }}$ edn., Sams, Indiana, 2002.

[6] C.-H. Liu, "On the design of OFDM signal detection algorithms for hardware implementation," IEEE Global Telecommunications Conference, vol. 2, pp. 596-599, Dec. 2003.

[7] H.-Y. Liu et al., "A 480Mb/s LDPC-COFDM-based UWB baseband transceiver," ISSCC Dig. Tech. Papers, pp. 444-446, Feb. 2005.

[8] A. Batra et al., "Preamble for a TFI-OFDM communications system," Patent US2004/0179507 A1, Sept. 16, 2004.

[9] W. C. Lim, B. Kannan, and T. T. Tjhung, "Joint channel estimation and OFDM synchronization in multipath fading," IEEE International Conference on Communications, vol. 2, pp. 983-987, Jun. 2004.

[10] W.-C. Chang et al., "An area and power efficient frame synchronizer for $480 \mathrm{Mb} / \mathrm{s}$ OFDM-based UWB system," IEEE International Symposium on VLSI Design, Automation and Test (VLSI-TSA-DAT), pp. 84-87, Apr. 2005. 